\title{
Two Children with CT-Scan Proven Brain Arachnoid Cyst and Different Clinical Manifestations Including Autism
}

\author{
Al-Mosawi AJ* \\ Department of Pediatrics, Children Teaching Hospital, Baghdad Medical City, Iraq
}

"Corresponding author: Aamir Jalal Al Mosawi, Department of Pediatrics, Children Teaching Hospital, Baghdad Medical City, Baghdad, Iraq, Tel: +964-7703930834; E-mail: almosawiaj@yahoo.com

\begin{abstract}
Background: Arachnoid cyst is a congenital disorder with variable clinical manifestations. Symptoms on the size and site of the cyst. Small cysts are generally asymptomatic. Clinical manifestations of arachnoid cyst include developmental and mental retardation, seizures, hemiparesis, headache, hydrocephalus, overactivity and other behavioral abnormalities. The association of arachnoid cyst with autism has been very rarely reported.

Patients and Methods: Two male children with CT-scan proven brain arachnoid cyst observed at the Children Teaching Hospital of Baghdad Medical City who have different clinical manifestations, and both have already been seen by more than two neurosurgeons and none of them recommended any surgical intervention.

Results: The first patient was seen at about the age of eight years. He had seizures that had already been controlled with sodium valproate, left spastic hemiparesis causing gait abnormalities and autism. Brain CT-scan showed arachnoid cyst in the left sphenoid 34mmx $16 \mathrm{~mm}$ in diameter, and periventricular leukodystrophy-like changes of signal abnormalities in the occipital area. The second boy was seen at the age of four years and nine months because of mental retardation, delayed speech and gait abnormalities caused by mild spasticity in the lower limbs. He also had seizures that had already been controlled with sodium valproate. Brain CT-scan showed large well-defined arachnoid cyst in left temporal fossa causing atrophy of the temporal lobe.

Conclusion: The rare association of arachnoid cyst with autism is reported and the association of mental retardation with left temporal arachnoid cyst is emphasized. Literature review suggested a possible role of acetazolamide in the treatment of large arachnoid cyst.
\end{abstract}

\section{Introduction}

Arachnoid cyst is a congenital cerebrospinal fluid filled cyst develops between the surface of the brain and the cranial base or on the arachnoid membrane which is one of the three meningeal layers that cover the brain and the spinal cord. Arachnoid cysts are a disorder. Symptoms on the size and site of the cyst. Small cysts are generally asymptomatic. Clinical manifestations of arachnoid cyst include developmental and mental retardation, seizures, hemiparesis, headache, hydrocephalus, overactivity and other behavioral abnormalities $[1,2,3,4]$. The association of arachnoid cyst with autism has been very rarely reported [5].

\section{Case Report}

Two male children with CT-scan proven brain arachnoid cyst observed at the Children Teaching Hospital of Baghdad Medical City who have different clinical manifestations, and both have already been seen by more than two neurosurgeons and none of them recommended any surgical intervention.

\section{Results}

The first patient was seen at about the age of eight years. He had seizures that had already been controlled with sodium valproate, left spastic hemiparesis causing gait abnormalities and autism. When the boy was first at the pediatric neuropsychiatry, he was not saying any word and displayed overactivity and autistics features dominated by lack of response to his name and absence of eye contact (Figure-1), and he also displayed repetitive movements. Brain CT-scan showed Figure-2 arachnoid cyst in the left sphenoid $34 \mathrm{~mm} \times 16 \mathrm{~mm}$ in diameter. There was also a periventricular leukodystrophy-like change of signal abnormalities in the occipital area. Cavum septum pelluciudum is considered a normal anatomical variation was seen. The posterior fossa structures were normal and there were no 


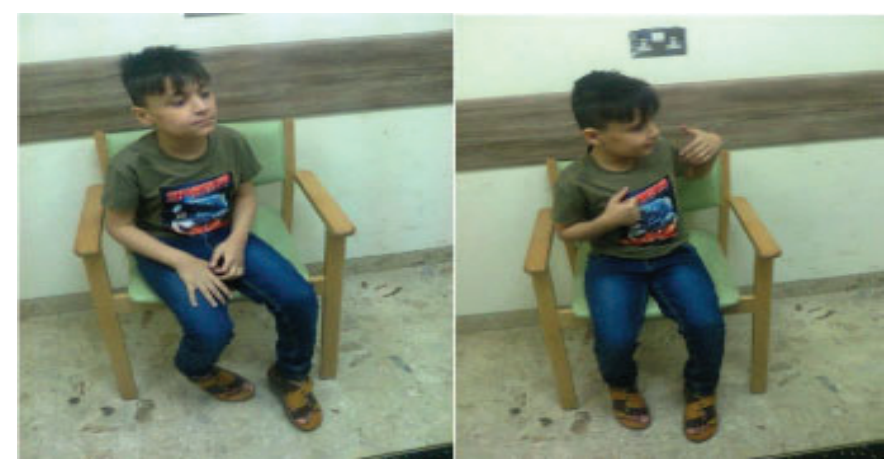

Figure 1: The boy showed autistics features dominated by lack of response to his name and absence of eye contact and he also displayed repetitive movements.

significant brain atrophic changes (Figure 2A, 2B, 2C, 2D, 2E, $2 \mathrm{~F}, 2 \mathrm{G}$ and $2 \mathrm{H})$.

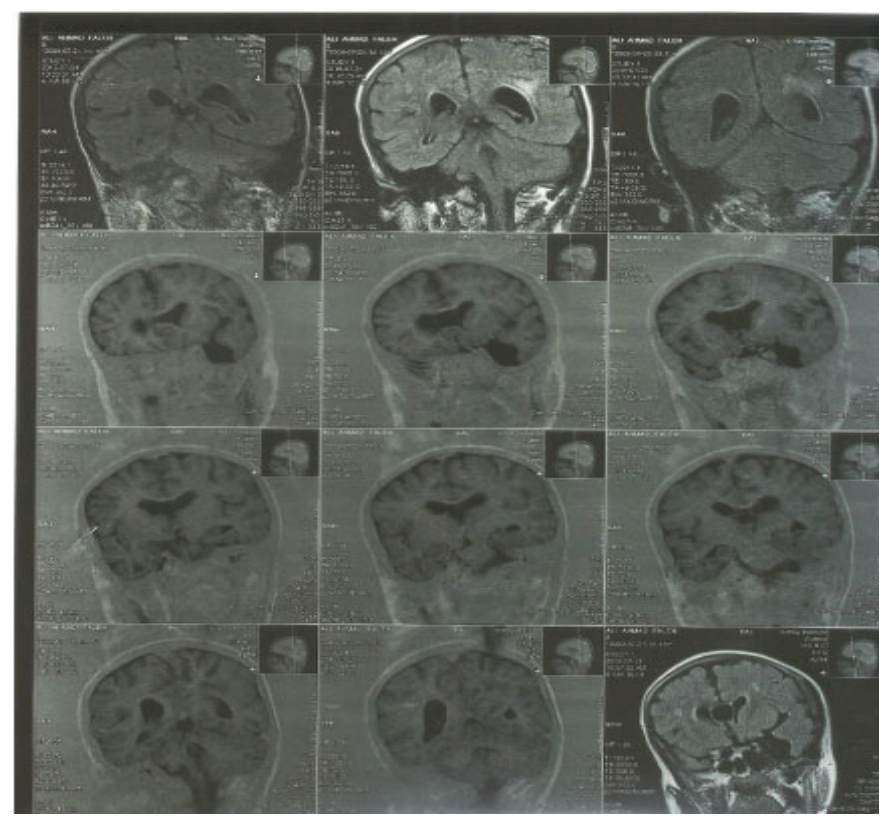

Figure 2A: Brain CT-scan showed arachnoid cyst in the left sphenoid, periventricular leukodystrophy-like changes of signal abnormalities in the occipital area and cavum septum pelluciudum

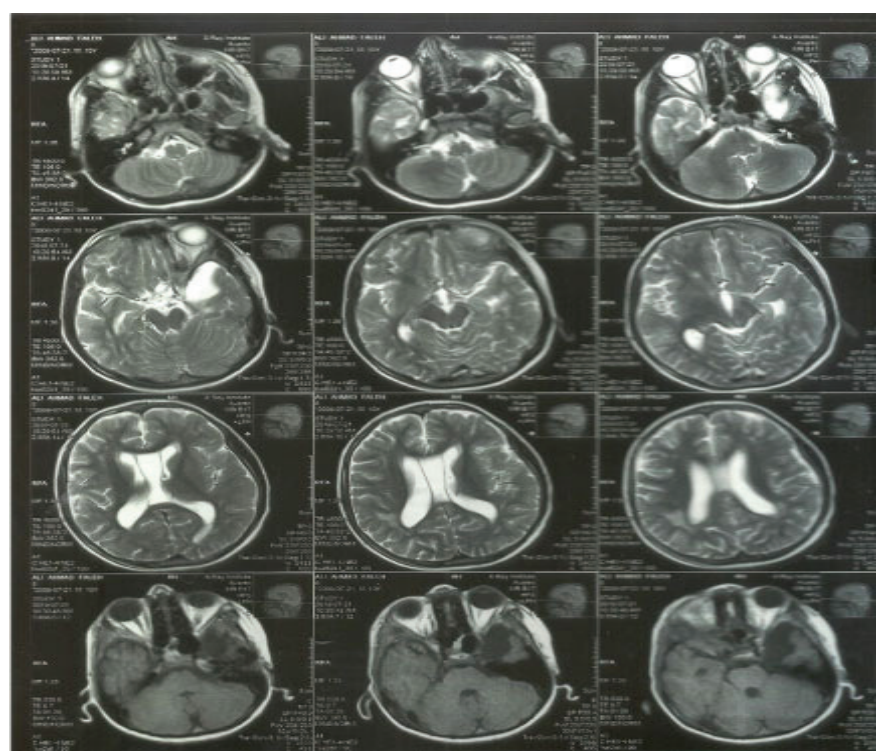

Figure 2B: Brain CT-scan showed arachnoid cyst in the left sphenoid, periventricular leukodystrophy-like changes of signal abnormalities in the occipital area and cavum septum pelluciudum

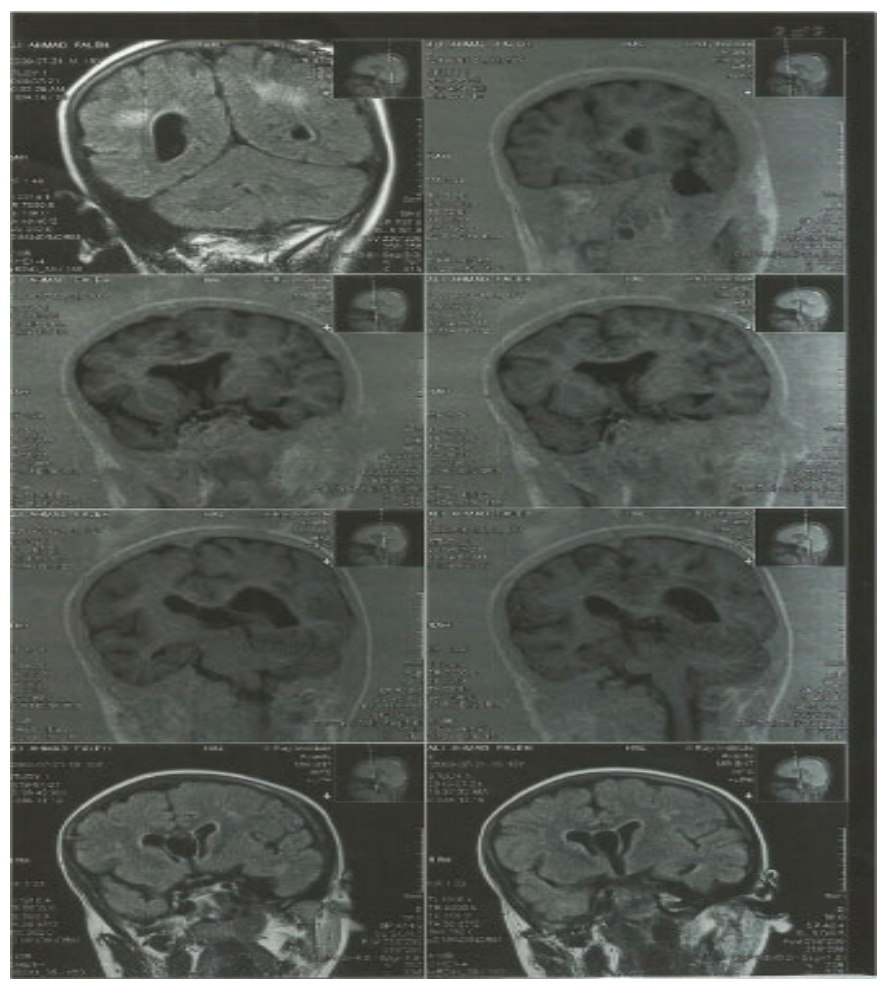

Figure 2C: Brain CT-scan showed arachnoid cyst in the left sphenoid, periventricular leukodystrophy-like changes of signal abnormalities in the occipital area and cavum septum pelluciudum

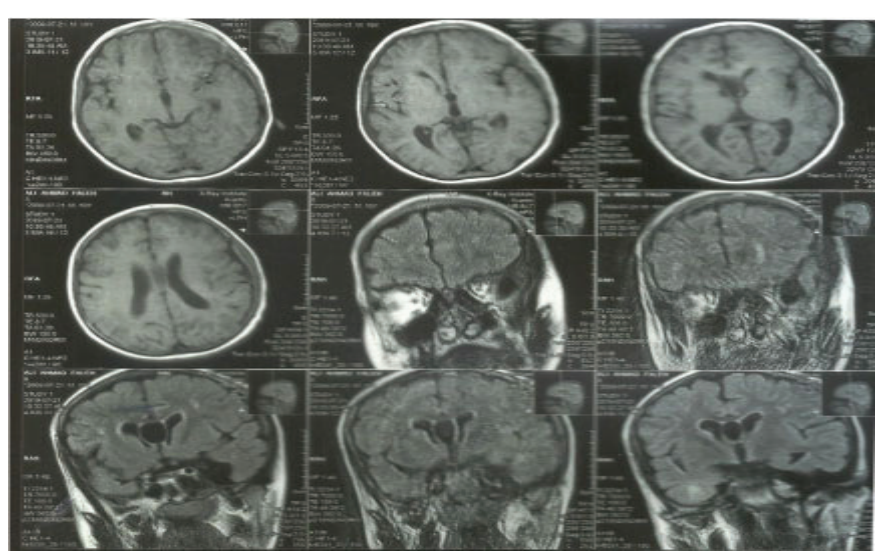

Figure 2D: Brain CT-scan showed arachnoid cyst in the left sphenoid, periventricular leukodystrophy-like changes of signal abnormalities in the occipital area and cavum septum pelluciudum

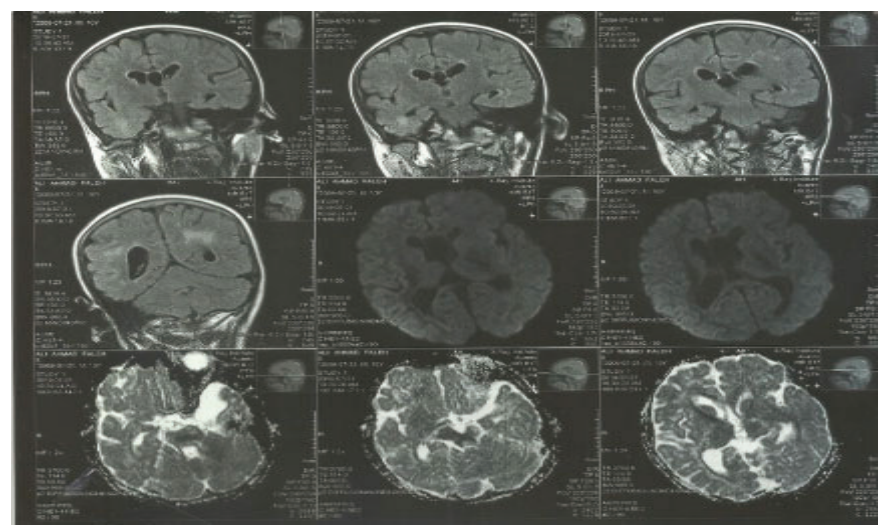

Figure 2E: Brain CT-scan showed arachnoid cyst in the left sphenoid, periventricular leukodystrophy-like changes of signal abnormalities in the occipital area and cavum septum pelluciudum 


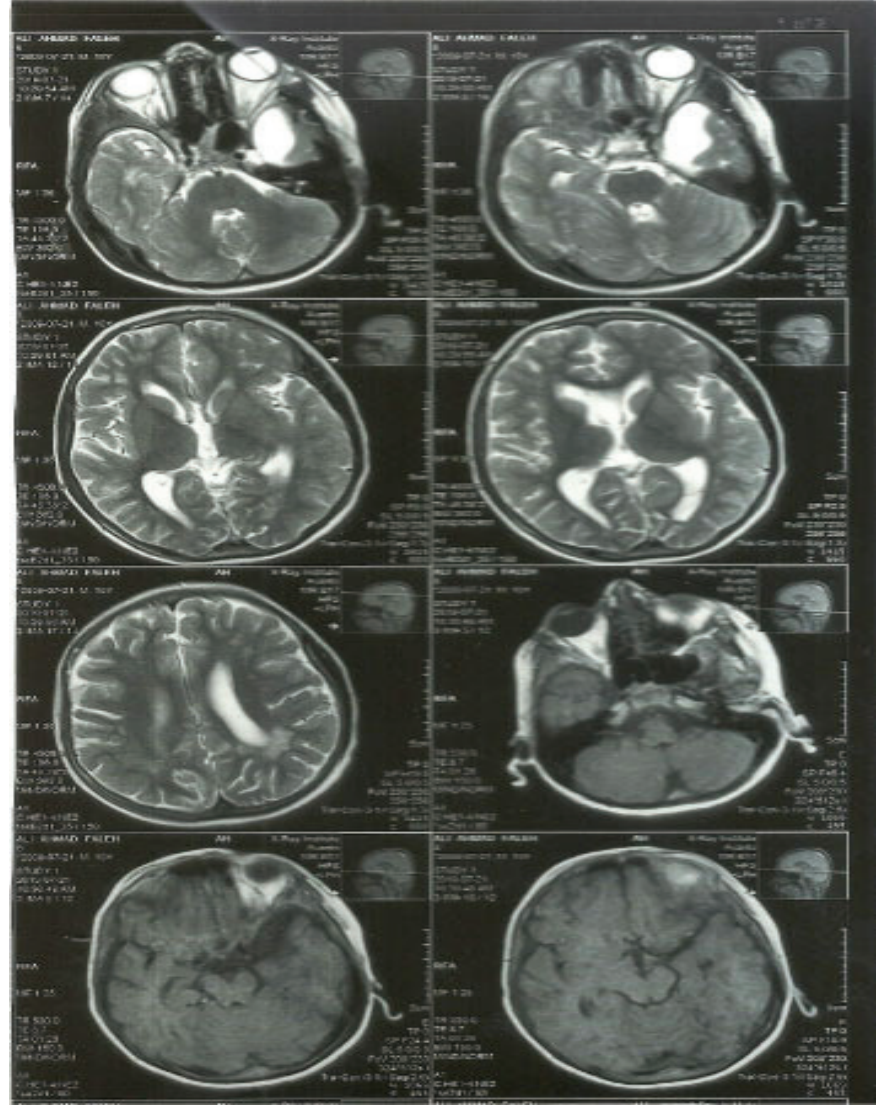

Figure 2F: Brain CT-scan showed arachnoid cyst in the left sphenoid, periventricular leukodystrophy-like changes of signal abnormalities in the occipital area and cavum septum pelluciudum

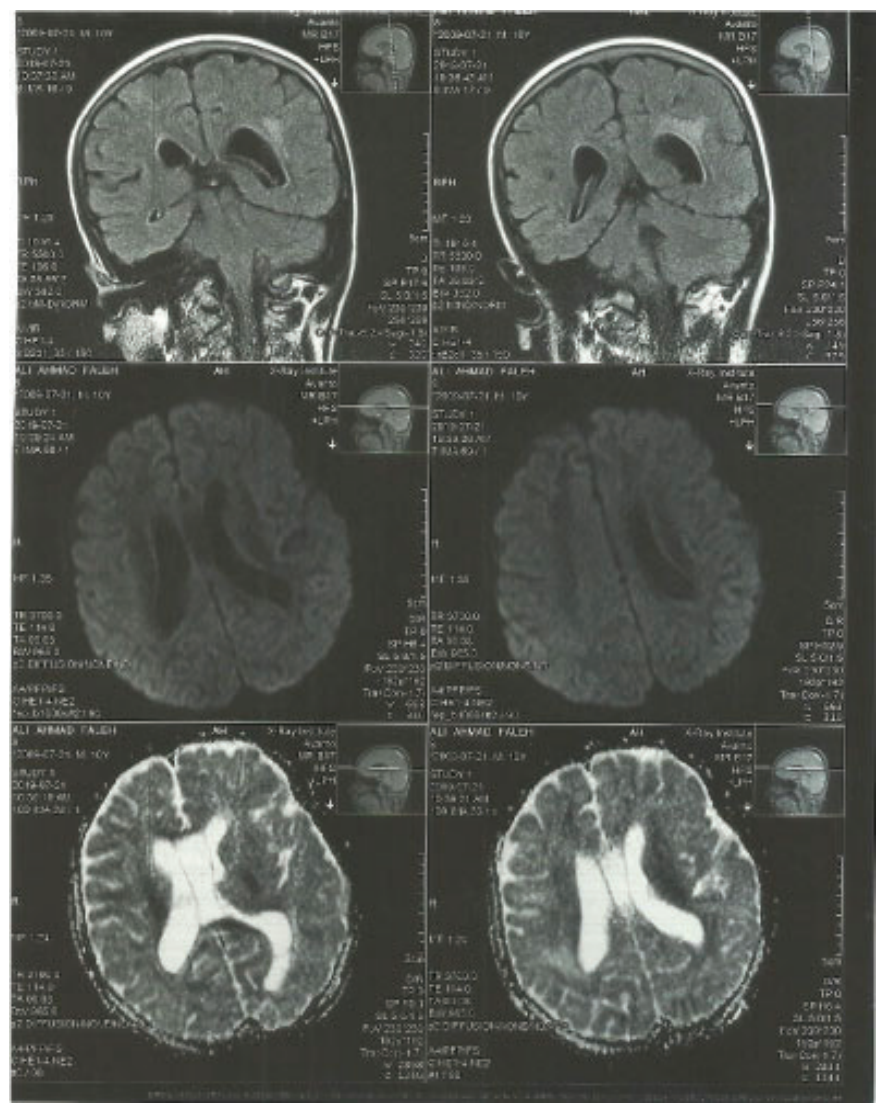

Figure 2G: Brain CT-scan showed arachnoid cyst in the left sphenoid, periventricular leukodystrophy-like changes of signal abnormalities in the occipital area and cavum septum pelluciudum

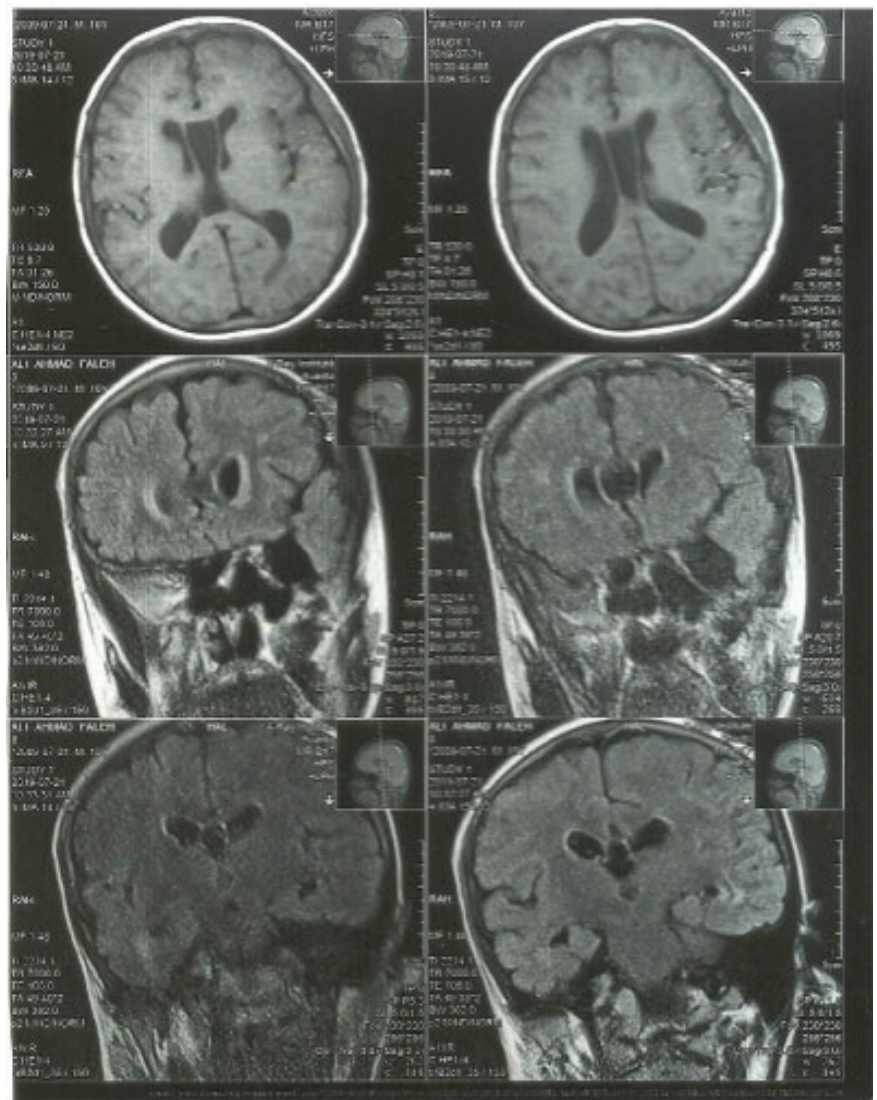

Figure 2H: Brain CT-scan showed arachnoid cyst in the left sphenoid, periventricular leukodystrophy-like changes of signal abnormalities in the occipital area and cavum septum pelluciudum

The second boy was seen at the age of four years and nine months because of mental retardation, delayed and gait abnormalities caused by mild spasticity in the lower limbs. He also had seizures that had already been controlled with sodium valproate. At the clinic, he was responding to his name and showed good eye contact, but he was overactive and tried to leave the examination room. However, he was eventually convinced to take a pen, but he couldn't copy a straight line (Figure-3). Brain CT-scan showed large well-defined arachnoid cyst in left temporal fossa causing atrophy of the temporal lobe.

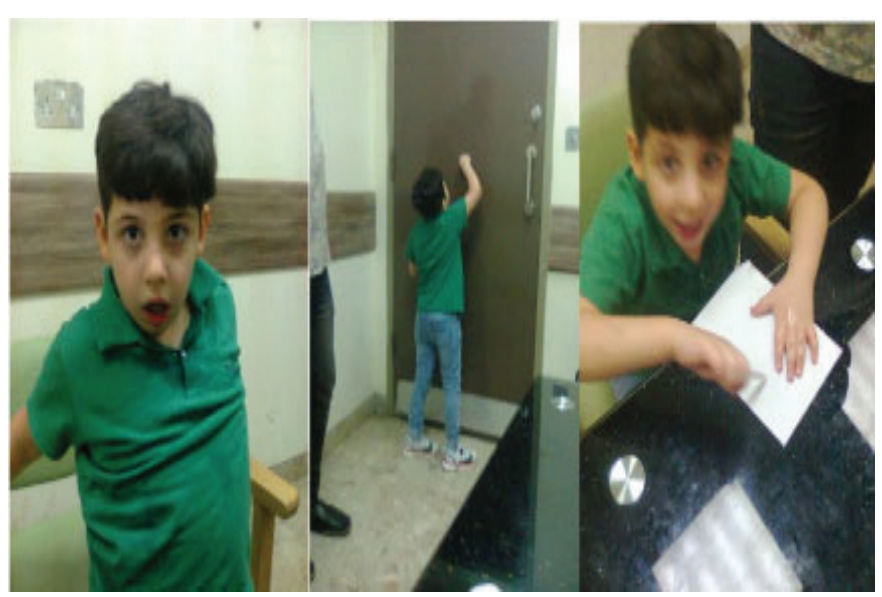

Figure 3: The was responding to his name and showed good eye contact, but he was overactive and tried to leave the examination room. However, he was eventually convinced to take a pen, but he couldn't copy a straight line 


\section{Discussion}

The association of arachnoid with mental retardation has been recognized as early as 1875 by Dr James Crichton Browne (November, 29, 1840-January, 31, 1938), a distinguished British neuropsychiatrist when he was the medical director of West Riding Asylum (Figure-4) [1], and more recent studied has shown an association of impaired cognition and arachnoid cysts particularly in the left temporal fossa [6], like the second patient in this paper.

The association of arachnoid cyst with autism has been very rarely reported [5]. Vaivre-Douret et al (2016) from France described a 6-year-old boy with pervasive developmental disorders-not otherwise specified and brain MRI showed a large arachnoid temporo-polar left cyst with a marked mass effect on the left temporal lobe. The authors reported that a neurosurgical intervention was associated with improvement in the symptoms.

Recently, Kershenovich and Toms (2017) from the United States thought that symptoms associated with arachnoid cyst may be related to the increased fluid pressure within the cyst on surrounding structures. They suggested that reducing the amount of fluid in the cyst or surrounding cerebrospinal fluid by with use of acetazolamide, a carbonic anhydrase inhibitor to reduce cerebrospinal fluid production, might mimic surgical decompression [7].

\section{Conclusion}

The rare association of arachnoid cyst with autism is reported and the association of mental retardation with left temporal arachnoid cyst is emphasized. Literature review suggested a possible role of acetazolamide in the treatment of large arachnoid cyst.

\section{Acknowledgement}

1.The author would like to express his gratitude for the patents of the children who willingly accepted publishing their photos.

2.The author owns the copyright of the sketch in figure-4.

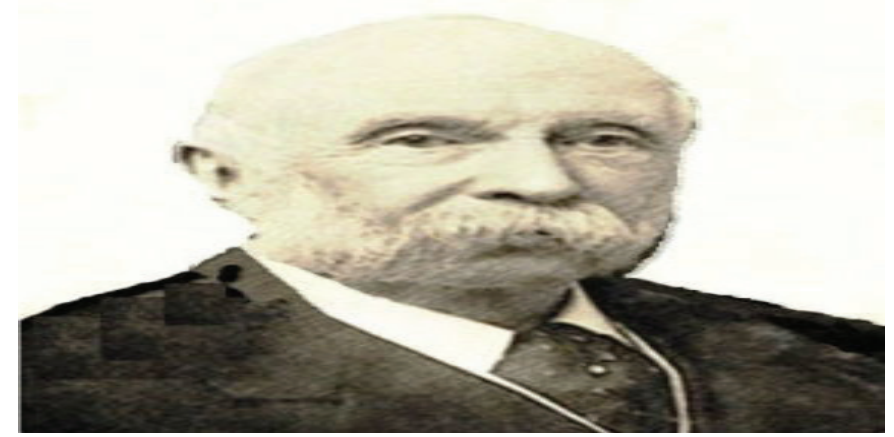

Figure 4: A sketch of Dr James Crichton Browne, a distinguished British neuropsychiatrist

\section{References}

1. Browne JC. Arachnoid Cysts. J Psychol Med Ment Pathol (London) Oct 1875; 1(2):167-181. PMID:28906874.

2. Cunningham DJ. A Large Sub-arachnoid cyst involving the greater part of the Parietal Lobe of the Brain. J Anat Physiol Jul 1879; 13(Pt 4):508-17. PMID: 17231284.

3. Heritage K. Suprasellar Arachnoid Cyst. Proc R Soc Med Nov 1937; 31(1):9-11. PMID: 19991305.

4. De Keersmaecker B, Ramaekers P, Claus F, Witters I, Ortibus E, Naulaers G et al. Outcome of 12 antenatally diagnosed fetal arachnoid cysts: case series and review of the literature. Eur J Paediatr Neurol Mar 2015;19(2):114-21. 27. PMID: 25599983.

5. Vaivre-Douret L, Boschi A, Cuny ML, Clouard C, Mosser A, Golse B et al. Left temporal arachnoid cyst and specific learning disorders associated with Pervasive Developmental Disorders-Not Otherwise Specified (PDD-NOS): contributions of an integrative neuropsychomotor, neuropsychological, psychopathological and neurosurgical approach about a case report in a child (François). Encephale Dec 2016; 42(6):582-588. PMID: 27644917[Article in French].

6. Wester K, Hugdahl K. Arachnoid cysts of the left temporal fossa: impaired preoperative cognition and postoperative improvement. J. Neurol. Neurosurg Psychiatry 1995; 59 (3): 293-8. PMID: 7673959.

7. Kershenovich A, Toms SA. The Acetazolamide Challenge: A Tool for Surgical Decision Making and Predicting Surgical Outcome in Patients with Arachnoid Cysts. J Neurol Surg A Cent Eur Neurosurg Jan 2017; 78(1):33-41. PMID: 27415592. 